\title{
XXXVI. A polarization flicker photometer and some data of theoretical bearing obtained with it
}

\author{
Herbert E. Ives
}

To cite this article: Herbert E. Ives (1917) XXXVI. A polarization flicker photometer and some data of theoretical bearing obtained with it , Philosophical Magazine Series 6, 33:196, 360-380, DOI: $10.1080 / 14786440408635650$

To link to this article: http://dx.doi.org/10.1080/14786440408635650

曲 Published online: 08 Apr 2009.

Submit your article to this journal $\asymp$

Џ Article views: 6

Q View related articles $\square$

Citing articles: 5 View citing articles 5 
XXXVI. A Polarization Flicker Photometer and some Data of l'heoretical Bearing obtained with it. By HERBent E. Ives*.

1. Introduction.

$$
\text { Synopsis. }
$$

2. Theory and Construction of a Polarization Flicker Photomoter.

3. Theory and Construction of a Mixture Photometer.

4. Critical Speed Relations for rarious relative Brightnesses of Two Compared Fields.

(a) Mean Brightness Constant.

(b) Meen Brightness Varied.

5. Measurement of the Brightness Difference Discrimination Fraction for Fluctuating Impressions.

6. Critical Speed Relations with a Difference of Colour between the Two Fields under Comparison.

7. Measurement of the Hue Difference Discrimination Fraction for Fluctuating Impressions.

8. Relative Values of Brightness and Hue Discrimination Fractions under Steady and Fluctuating Conditions.

9. Hffect of Choice of Speed on Flicker Photometer Settings.

10. Summary.

\section{Introduction.}

T previous patpers by the present writer and Mr. F. F. 1 Kingsbury on "The Theory of the Flicker Photometer" $t$, emphasis was laid on the desirability, from both the practical and theoretical sides, of a flicker photometer in which the transition from one field to the other should be gradual and according to some simple mathematic relation. Practically the advantage is in eliminating mechanical flicker, due to dividing edges, which can introduce spurious effects. Theoretically the advantage is that this kind of transition, especially if it can be represented by the simplest periodic function-the cosine carve-can be handled mathematically $\ddagger$.

The theory developed by us was based on a stimulus represented by a cosine curve. The verification of the theory by experiment, which was sought in work with flicker photometers with abrupt transition, while very satisfactory, was incomplete quantitatively in several details. We gave reasons for believing that the outstanding discrepancies were ascribable to the difference between the type of

* Communicated by the Author.

+ "Theory of the Flicker Photometer," Ives \& Kingsbury. Phil. Mag. Nov. 1914, p. 708; A pril 1916, p. 290.

\pm Other transitions can of course be represented mathematically; the difficulty is in solving the equations resulting when the diffusivity conditions are introduced. 
flicker photometer theoretically postulated and that actually used.

In the present paper a flicker photometer is described in which the transition from one light to another is exactly of the character handled in the theory. It has been possible with this photometer to verity accurately the predictions of the theory. Measurements of the visual constants have been made, and, as a consequence, the complete behaviour of this type of equal exposure flicker photometer can now be represented quantitatively by a set of equations derived from the physical theory of conduction.

\section{Theory and Construction of a Polarization Flicker Photometer.}

The polarization flicker photometer is a combination of a double-image prism with a rotating Nicol prism. Two images of each half of the photometric field are formed by the double-image prism, and the dimensions of the apparatus are so chosen that the horizontally polarized image of one half is superposed on the vertically polarized image of the other. The Nicol prism as it rotates alternately extinguishes each image, the one fading gradually into the other, with entire absence of any mechanical edge effect.

Fig. 1.

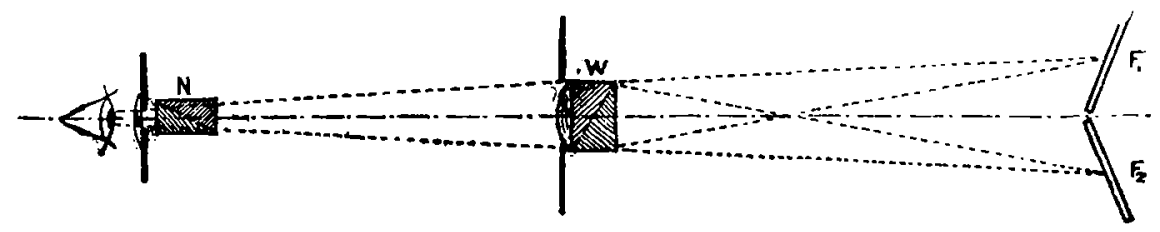

Polarization flicker photometer: $\mathbf{N}$, rotating Nicol prism ; $\mathbf{W}$, doubleimage prism; $F_{1}$ and $F_{2}$, two halves of photometer field.

Fig. 2.

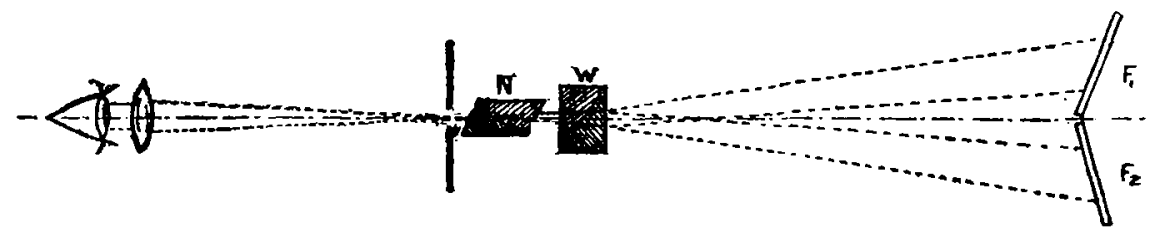

Alternative arrangement of polarization flicker photometer.

Figs. 1 and 2 show two constructions. In fig. 1 the Nicol prism is placed at the eye. When so used the nicol must be of the type in which the faces are perpendicular to the beam

Phil. Mag. S. 6. Vol. 33. No. 196. April 1917. 2 B 
of light, otherwise the whole field will oscillate with the rotation of the prism. In fig. 2 the nicol is placed away from the eye and beyond the diaphragm which limits the field. In this arrangement the nicol may be of the obliquefaced type. The apparatus actually used was arranged as in fig. 2, because of the kind of nicol prism available. The distances were such that the photometric field had an angular aperture of $1 \frac{1}{2}$ degrees. The artificial pupillary aperture was $3 \mathrm{~mm}$. in diameter.

The theory of the polarization flicker photometer is simple. Let $2 \mathrm{I}_{0}$ be the brightness of the image polarized in one plane, then the brightness of the image when the nicol is rotated through the angle $\phi$ from that plane is

$$
2 \mathrm{I}_{0} \cos ^{2} \phi \text {. }
$$

Now

$$
2 \mathrm{I}_{0} \cos ^{2} \phi=\mathrm{I}_{0}(1+\cos 2 \phi) \text {. . . . }
$$

If we put $2 \phi=\omega t$, the right-hand member of (1) becomes the right-hand member of equation (1) in the first theoretical paper, that is, we have exactly the type of stimulus there postulated.

\section{Theory and Construction of a Mixture Photometer.}

In the discassion of colour flicker it was shown that the action of the flicker photometer is to form a mixture of the two colours under measurement. The speed at which it is necessary to run the flicker photometer in order that there shall be no colour flicker is that at which the hues of the extreme mixtures differ less than the least perceptible hue difference. The relationsilip of this hue difference to the least perceptible brightness difference and the relationship of both of these to the corresponding quantities for steadily viewed juxtaposed fields, are crucial points in flicker photometer theory.

In order to be prepared for hue difference perception measurements the photometer "head," if it may be so callod, was made in such a way that the light from the two sources under comparison could be mixed in any desired proportions. The apparatus is shown diagrammatically in fig. 3 . It consists of two translucent (flashed opal) glasses, arranged, one over the other, to turn by means of gears in opposite directions, about a common axis parallel to their faces. This dovice is set up at the intersection of two photometer tracks, standing at right angles to each other, and is viewed from the rear. If used for equality of brightness observations a biprism is employed to juxtapose images of the 
two glasses. If used with the polarization apparatus the divergence of the double-image prism-beams easily clears the gap between glasses. A thin metal screen is placed in this gap to prevent illumination of one glass by the other, and the edges of the glasses are coated with opaque black paint.

Fig. 3.

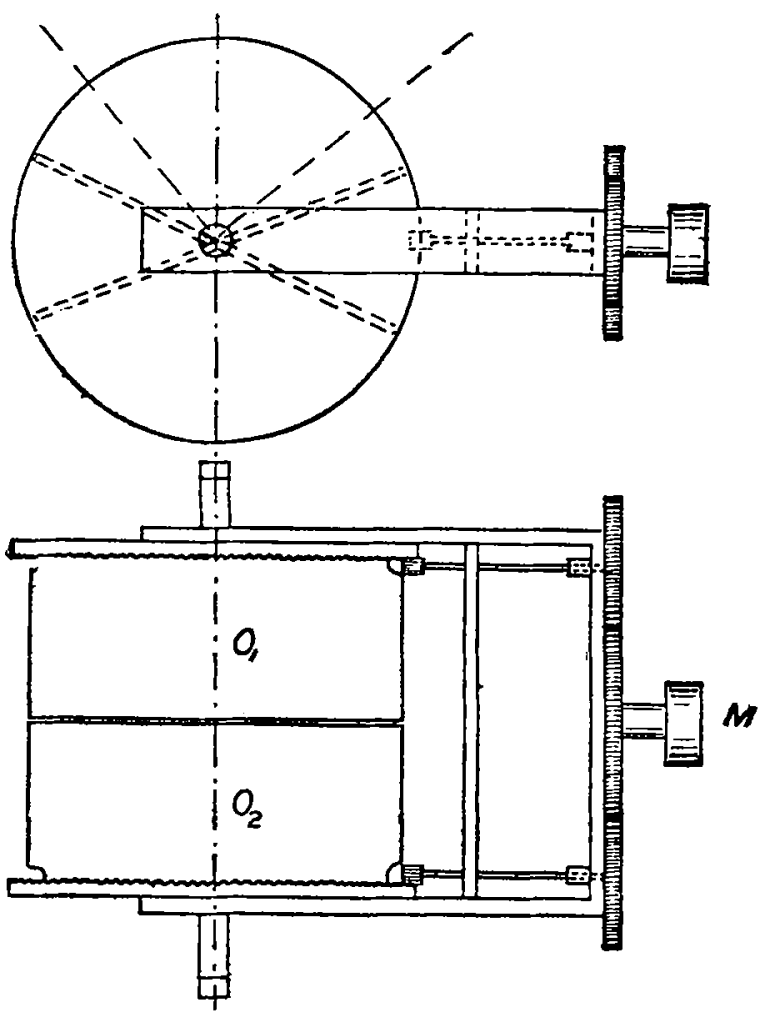

Hixture photometer : $\mathrm{O}_{1}$ and $\mathrm{O}_{2}$ are opal glasses turned in opposite directions by the milled head $M$.

With the glasses turned at right angles to each other and normal to the two photometer tracks the two glasses form the two halves of an ordinary equality photometer field. When turned at other angles various mixtures of the two lights are obtained. The brightness of each glass at various angles due to illumination by each light alone was carefully determined by measurements with a portable photometer. In 
fig. 4 is exhibited the brightness of each glass due to illumination by a light source on one track alone, as the glasses are rotated. The sum of the two is calculated in order to facilitate moving the light sources, so that the mean brightness of the two glasses may be maintained constant as their relative values are changed by their rotation.

Fig. 4.

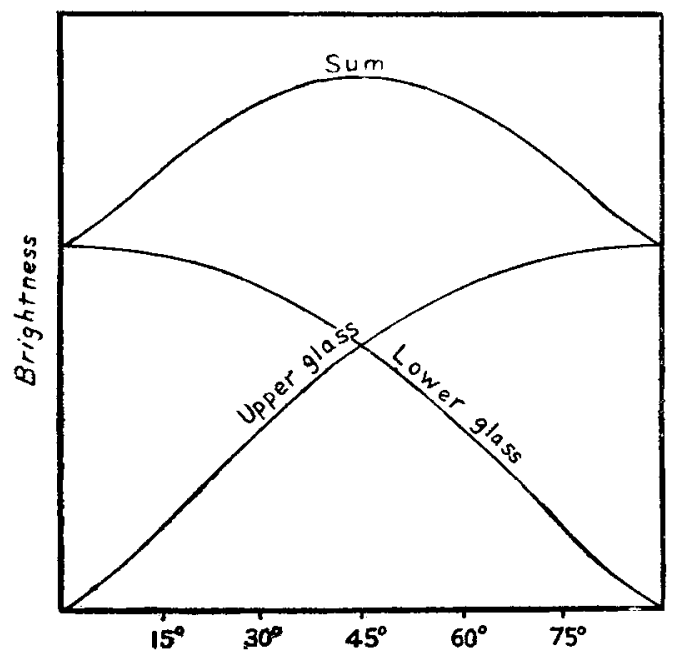

Relative brightness of upper and lower glasses of mixture photometer due to a single light source, for various angles of incidence of light on lower glass.

Using one light source, this device permits the comparison of fields of all degrees of relative brightness, but of the same colour. Using two light sources of different colour, but the same intensity, the device makes possible the comparison of fields of all hues intermediate between the two, formed by their mixture.

As a precautionary measure, the light transmitted by the two opal glasses was tested for any difference in polarization which would affect the setting of the polarization flicker photometer, but nothing of the sort was found.

4. Critical Speed Relations for various relative Brightnesses of Two Compared Fields.

(a) Mean Brightness Constant.

In the second of the papers on the theory the following equation is derived for the critical speed of disappearance of 
flicker between two fields of unequal brightness :

$$
\omega=\frac{2\left[\log \frac{2}{\delta}+\log _{\mathrm{I}_{1}-\mathrm{I}_{2}}\right]^{2}}{\overline{\mathrm{X}}(\log e)^{2}}, \ldots . .
$$

where

$$
\begin{aligned}
\omega & =\text { critical speed, } \\
\delta & =\text { brightness discrimination fraction, } \\
\mathrm{I}_{1} \text { and } \mathrm{I}_{2} & =\text { brightness of the two fields, } \\
\mathrm{K} & =\text { diffusivity, } \\
\mathrm{X} & =\text { depth of penetration. }
\end{aligned}
$$

If we assume that $\mathrm{K}$ is a function of the mean brightness, we can, by holding the latter constant as $I_{1}$ and $I_{2}$ are varied relatively to each other, make a test of the theory, in so far as it assumes $a$ diffusivity apart from any question as to how this varies with intensity. Fig. 1 of the paper referred to shows critical frequencies for various values of $I_{1}$ and $I_{2}$ under these conditions, which were not attainable experimentally with the apparatus then available.

With the new apparatus just described the exact conditions for this test are met. In fig. 5 are shown the results of two extended sets of measurements of eritical speeds for varying ratios of two fields, with the mean brightness held constant. The upper points were obtained at a relatively high, the others at a relatively low, illumination. The full lines are graphs of the equation (2), with a value $\delta$ of $\cdot 001$, using the values of $\frac{X^{2}}{\bar{K}}$ solved for from the end points. It is clear that the theory is very beautifully borne out.

(b) Mean Brightness Varied.

The second theoretical paper gives in equation (6) a relation for the critical speeds at different illuminations, which upon inserting the complete expressions for the constants becomes

$$
\omega=\frac{\left[\log \frac{2}{\delta}+\log \frac{\mathrm{T}_{1}-\mathrm{T}_{2}}{\mathrm{~T}_{1}+\mathrm{I}_{2}}\right]^{2}}{\left(\log _{\delta}^{2}\right)^{2}}\left(a \log \mathrm{I}_{\mathrm{A}}+b\right), \ldots .
$$

where $\mathrm{I}_{\mathbf{\Delta}}$ is the mean brightness, and $a$ and $b$ are the constants (as determined by experiment) applying to the limiting case of light alternated against darkness. It follows from this equation that, critical speeds for the limiting case being 
represented by a straight line when plotted against the logarithm of the intensity, critical speeds for other ratios of the fields should plot as straight lines lying below and

Fig. 5.

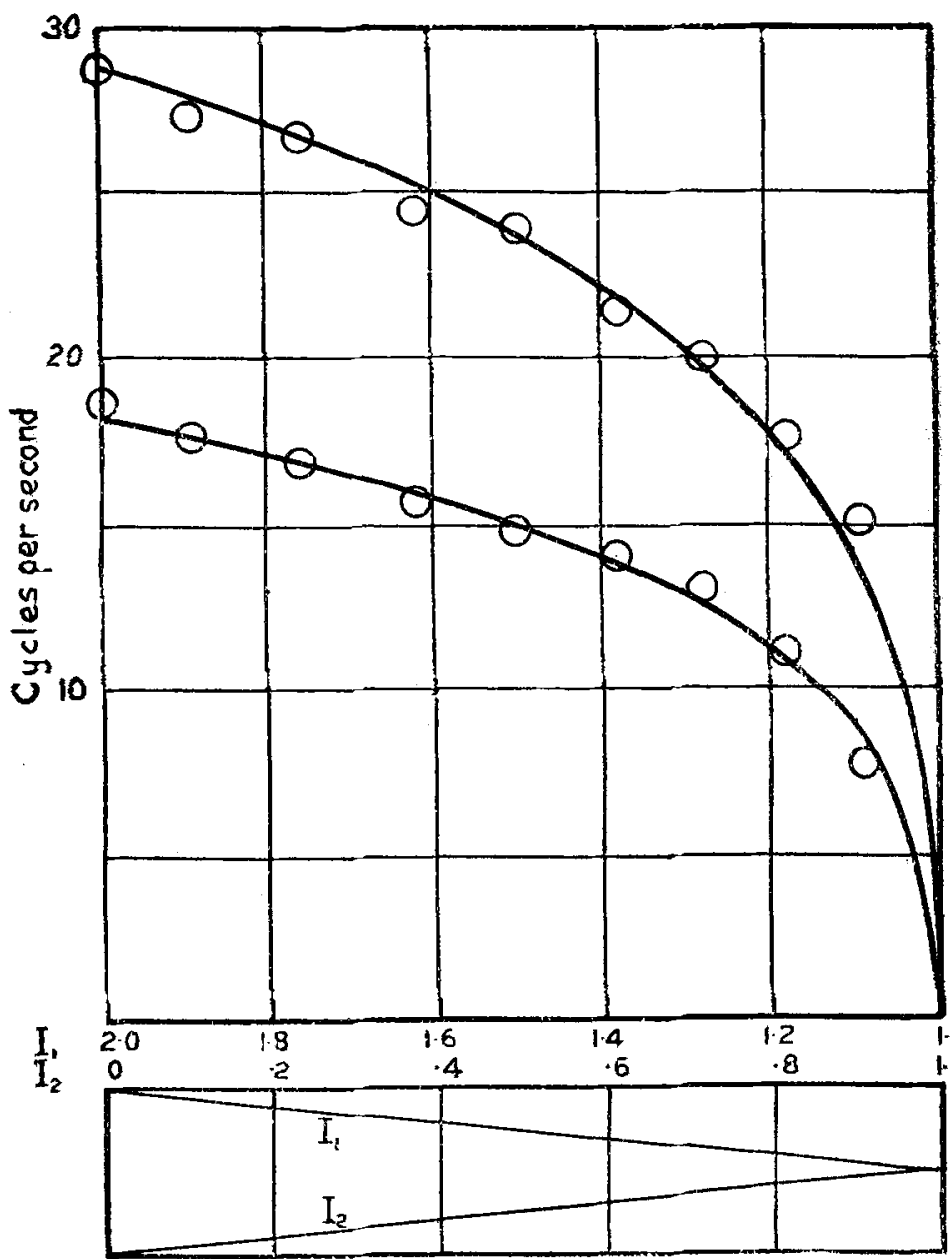

Critical frequencies for various ratias of brightness of two compared fiolds. Full lines, calculated curves for $\log \delta_{B}=-30$.

inclined to this at such angles that all will meet at a point on the axis of abscissæ. In our previous experimental work we did not get satisfactory confirmation of this equation. 
Fig. 6 shows experimental values obtained to test the equation, using the new photometer. It will be seen that the theory is fully confirmed. Equation (3) is hence the general expression, of which the relation for light against darkness $\left(I_{2}=0\right)$, as found by Porter, is a special case.

Fig. 6

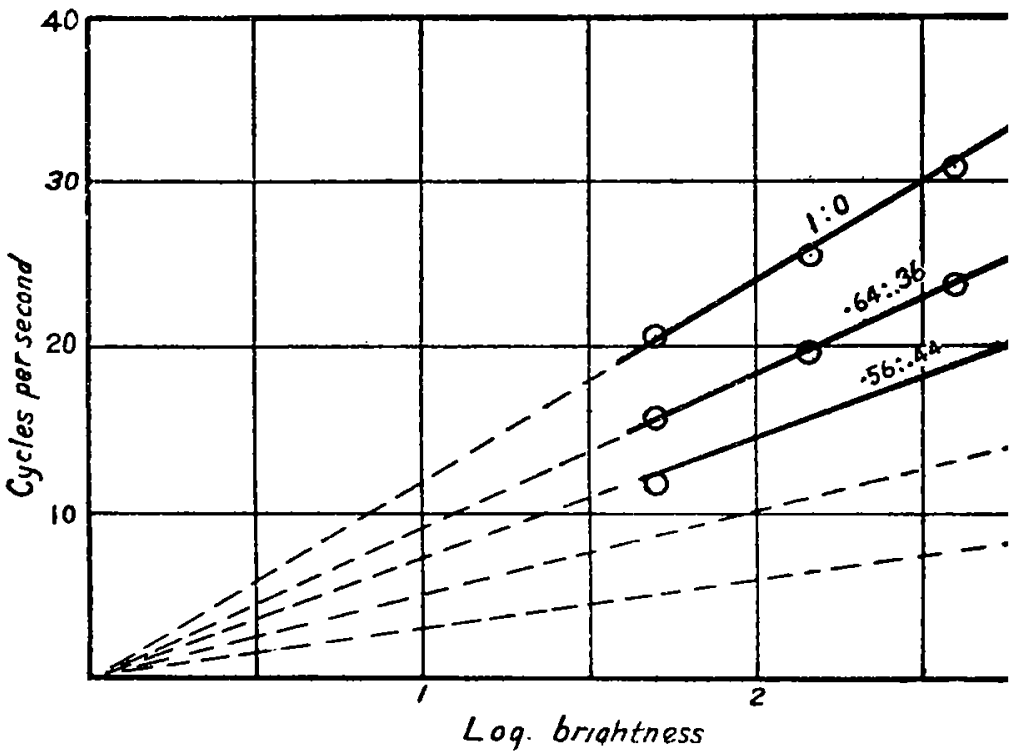

Critical speed brightness relations for variou ratios of brightness of two fields.

The value of $\delta$ called for by these data is abont $\cdot 0001$, which is much smaller than the figure fitting the data of the previous section. The measurement of $\delta$ and its characteristic of variability call for separate discussion.

\section{Measurement of the Brightness Diffierence Discrimination Fraction for Fluctuating Impressions.}

A determination of the critical speeds for two different ratios of the fields furnishes all the necessary data for finding the value of $\delta$.

Denoting the two different critical speeds and brightness ratios by the subscripts $a$ and $b$, we obtain from (3)

$$
\log \delta=\log 2-\frac{\log \left(\frac{I_{1}-I_{2}}{\mathrm{I}_{1}+I_{2}}\right)_{a}-\sqrt{\frac{\omega_{a}}{\omega_{b}}} \log \left(\frac{I_{1}-I_{2}}{I_{1}+I_{2}}\right)_{b}}{\sqrt{\frac{\omega_{a}}{\omega_{b}}-1}} .
$$


If for one of the brightness ratios is taken that of light against darkness the corresponding logarithmic term drops out, being zero. Measurements of the critical speeds for the ratios $1: 0$ and $\cdot 34: \cdot 66$ were made by the writer on several different occasions during the work, each consisting of four to six alternated series of ten settings each, from which the following values of $\log \delta$ were found :-

$$
\begin{aligned}
\log \delta= & -3 \cdot 0 \\
& -4 \cdot 2 \\
& -3 \cdot 7 \\
& -4 \cdot 2 \\
& -5 \cdot 0
\end{aligned}
$$

These figures, all obtained for the same mean brightness, show that $\delta$ varies over quite wide limits, for these variations represent rather bigger differences in the critical speed ratios than can be ascribed to the lack of precision of the determinations, considerable though this is. There is clear indication of a progressive decrease in $\delta$ during the progress of the work.

In order to secure additional light on this point, similar measurements were secured from other observers who had not been working with the apparatus.

Their results are as follows :-

$$
\left.\begin{array}{rl}
\log \delta= & -4 \cdot 2(\text { K. K.) } \\
& -3 \cdot 0(\text { ( } . \text { F. K. }) \\
& -3 \cdot 2 \text { (F. F. K. }) \\
& -3 \cdot(\text { E. R. M.) }
\end{array}\right\} \text { several days apart, }
$$

indicuting that the fraction varies from individual to individual, as well as from time to time with any one observer.

The mean value of all these determinations is

$$
\log \delta=-3 \cdot 74 \text { or } \delta=\cdot 00018 \text {. }
$$

Remembering that the Fechner fraction, as ordinarily understood and determined for juxtaposed fields, is of the order of magnitude of 1 per cent., this value of $\delta$ appears extraordinarily low. It is well known, however, that the greatest sensibility to small brightness difference is obtained when the compared fields are most closely juxtaposed, but even then the comparison must be made between impressions received by different retinal elements. In the case of successive impressions the comparison is made of impressions on the same element, and there is notbing inherently improbable in the sensibility to such impressions being higher than the greatest sensibility attainable by juxtaposition. It 
is to be noted, however, that this high sensibility, located as it is in the ultimate receiving apparatus, can never be utilized to its full value for the detection of small fluctuations in the stimulus. Because of the damping effect of the channel through which the impression of the fluctuating stimulus is transmitted, only very considerable fluctuations retain any amplitude large enough to be detected. In other words, the very small value of the brightness discrimination fraction of the ultimate receiver for successive impressions is all that makes possible our appreciation of ordinary fluctuations in the stimulus. If this had the value of the ordinary Fechner fraction we could tolerate much lower frequencies of light fluctuation than we now do. On the other hand, if we could stimulate the ultimate receiver directly, without the interposition of the present transmitting channel, it would be necessary to use rather high speeds of alternation to make flicker vanish. Upon substituting different values of $\delta$ in equation (4) of the second paper on the theory, the effect on $\omega$ is given numerically. A value of 01 for $\delta$ in place of 00018 about halves the critical speed.

\section{Critical Speed Relations with a Difference of Colour between the Two lields under Comparison.}

It follows from the treatment, in the two theoretical papers, of colour flicker and of brightness flicker with unequally bright fields, that critical speeds should follow the same law in the case of two coloured fields as with two unequally bright fields, up to the point of the appearance of colour flicker. The latter interposes a bar to the progressive decrease of speed to zero which occurs at the equal brightness point for fields of the same colour. Detailed discussion of the statement just made may be avoided by reference to the diagrammatic insert to fig. 7. Here the two fields under comparison are supposed to be of different colour. The lefthand end of the figure represents the condition with one colour field alone exposed, and, as indicated below the larger figure, the brightness of this field is progressively decreased while the other field is increased in such manner that their sum remains constant. All points below the two critical frequency curves are in the region of brightness flicker. The curve which is drawn convex upward, with its maximum at the equal brightness point, is taken from the first theoretical paper and is the upper limit to the colour flicker region, or region in which the hue difference for successive phases of the flicker photometer is greater than the least perceptible. 
No setting can be made at a lower speed than that fixed by the intersection of these curves. The actual observation of a flicker-limit curve such as that indicated by the small diagram, with its very definite truncation of the brightness flicker curves, should constitute a strong argument for the correctness of the line of reasoning followed.

For the experimental test of these relations, red and green lights were taken as furnished by two point source 100 watt tungston lamps shining through red and green glasses of fairly narrow spectral transmissions. The two lamps were operated in parallel, one terminal of each going to the battery, the other terminals being connected to the opposite ends of a resistance, the sliding middle point of which was joined to the other battery terminal. By moving the sliding contact the intensity of one lamp increased while the other decreased, and vice versa, this constituting the best way of operating a flicker photometer, as previously pointed out. The first operation in this experiment consisted in finding, by the flicker photometer criterion, the equal brightness voltages for the lamps through their coloured glasses, each illuminating one of the opal glasses, placed normal to it, and these voltages were used throughout the work with them.

In fig. 7 are shown the critical frequencies obtained on carrying through the entire series of comparisons of the two coloured fields, the various relative intensities being obtained by moving the two lamps to various calcnlated positions on the photometer bar. It will be seen that the points follow the curves calculated from equation $(2),(\delta=0002)$ right down to the sharp intersection of the colour flicker limiting curve and then follow this across from one brightness flicker limiting curve to the other. As they should be, the critical frequencies at the end points are not exactly the same for two coloured lights equal by the flicker photometer criterion. The experimentally found relationships are in every respect in agreement with those indicated by the theory.

\section{Measurement of the Hue Inifference Discrimination Fraction for Fluctuating Impressions.}

Exact determination of the speed at which colour flicker appears, which is made possible by the new flicker photometer, opens the way to the measurement of the just perceptible hue difference corresponding to the brightness difference fraction discussed in section 5 .

In the first theoretical paper it is shown in equation (9) and its discussion, that if two coloured fields $R$ and $G$ of 
Fig. 7 .
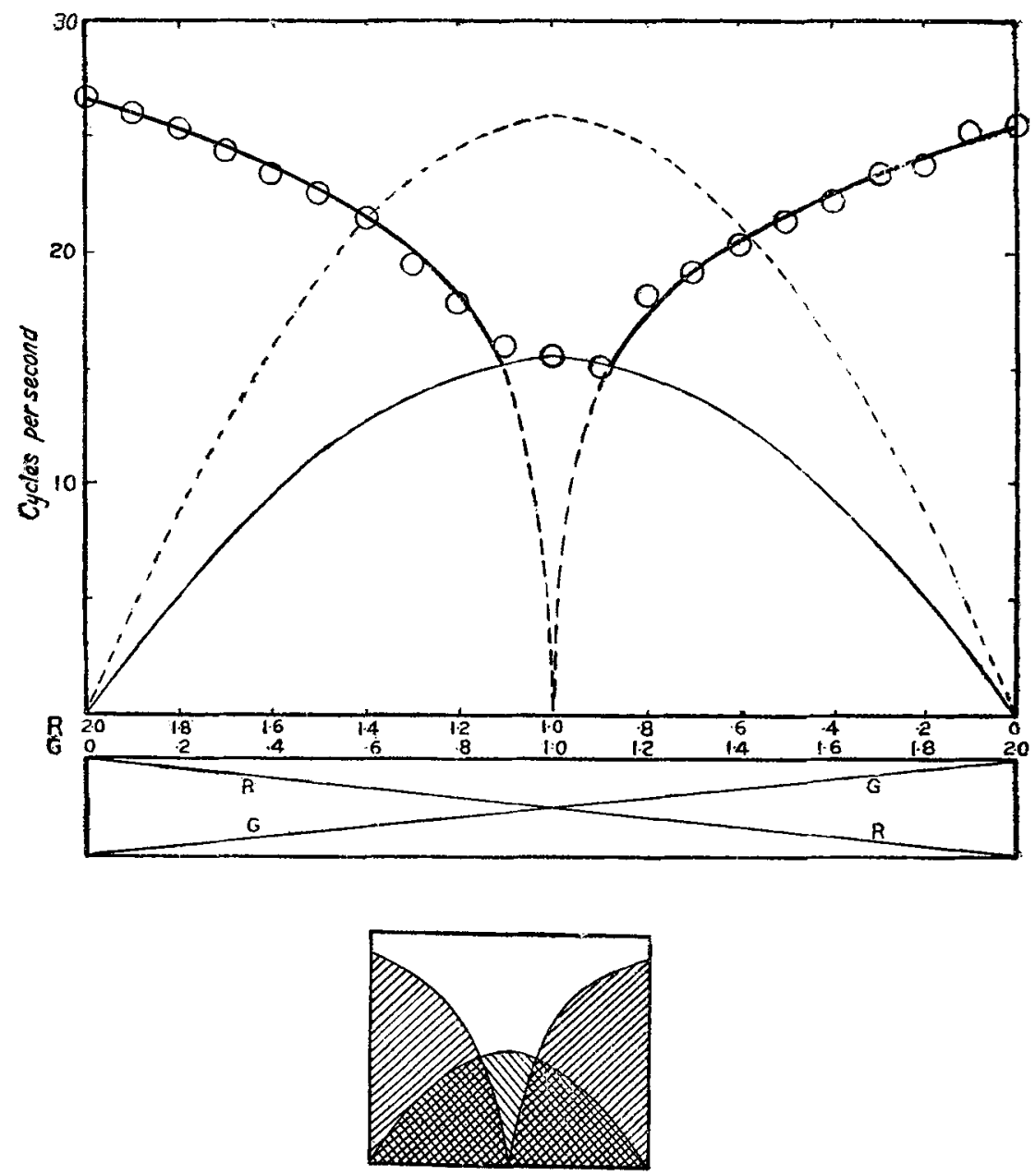

Brightness flicker

Color flicker

Critical speed relations with a difference of colour between the two fields. Lower, diagrammatic ; upper, experimental. Upper dashed curve, limit of colour flicker region if fluctuating hue discrimination fraction maintained its steady observation ratio to brightness. discrimination fraction. 
equal brightness are alternated in the flicker photometer, the ratio of $R$ to $G$ at one extreme phase is given by

$$
\frac{\mathrm{R}}{\mathrm{G}}=\frac{\left(1+e^{\frac{\mathrm{X}}{\sqrt{2 \mathrm{~K}_{1}}} \sqrt{\omega}}\right)}{\left(1-e^{-\frac{\mathrm{X}}{\sqrt{2 \mathrm{~K}_{2}}} \sqrt{\omega}}\right)}
$$

and at the other phase by the similar expression with the + and - signs interchanged.

Before detailed discussion of this it is desirable to digress in order to develop the idea of a hue scale by which a numerical value can be given to any hue, and in particular the idea of a hue discrimination fraction will be developed and used.

Let us imagine the two juxtaposed fields of the mixture photometer illuminated by two equally bright but differently coloured light sources $\mathrm{R}$ and $\mathrm{G}$. When the two opal glasses stand at right angles to each other and normal to each light source they are of the colour of these latter, and show their maximum colour difference. As they are turned towards parallelism they are illuminated by mixtures of the two colours and become progressively more like in hue, until at the position of exact parallelism they are the same. On turning further they diverge in bue until at the end position they show the same difference as at first, but in the opposite direction. Now if we consider these hues as forming a continuous series, they may be described and given a numerical value in terms of the proportion of one of the end colours present in the mixture forming any hue. If, for instance, we identify the hue by the amount of $R$ in it, we have that the hue, $H$, of the mixture in which the two colours are present in the ratio $R_{H}: G_{H}$ is

$$
\frac{R_{H}}{R_{H}+G_{H}} \text {. }
$$

A hue difference $\mathrm{H}_{1}-\mathrm{H}_{2}$ is then

$$
\frac{\mathrm{R}_{\mathrm{H}_{1}}}{\mathrm{R}_{\mathrm{H}_{1}}+\mathrm{G}_{\mathrm{H}_{1}}}-\frac{\mathrm{R}_{\mathrm{H}_{2}}}{\mathrm{R}_{\mathrm{H}_{2}}+\mathrm{G}_{\mathrm{H}_{2}}} \text {, . . . . . }
$$

and this is the fractional uifference

$$
\frac{\mathrm{H}_{1}-\mathrm{H}_{2}}{\frac{\mathrm{H}_{1}+\mathrm{H}_{2}}{2}} \cdot \text {. . . . . . }
$$


If this hue difference is the least perceptible one, then we define the hue difference discrimination fraction by relation (7). We shall use the symbol $\delta_{H}$ for this fraction. It will be noted that this definition is exactly parallel to the definition of the brightness discrimination fraction, which is

$$
\frac{I_{1}-I_{2}}{\frac{\Gamma_{1}+I_{2}}{2}} \cdot \text {. . . . . . }
$$

This will be symbolized by $\delta_{B}$ hencetorth, to avoid confusion.

Applying now (6) and (7) to (5), we have that the hue at one phase is

$$
\mathrm{H}_{1}=\frac{1+e^{-\frac{\mathrm{X}}{\sqrt{2 \mathrm{~K}_{1}}} \sqrt{\omega}}}{2} ;
$$

at the other phase it is

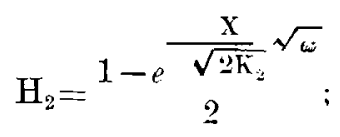

from which

$$
\delta_{\mathrm{H}}=\frac{\mathrm{H}_{1}-\mathrm{H}_{2}}{\frac{\mathrm{H}_{1}+\mathrm{H}_{2}}{2}}=\frac{i\left[e^{\left.-\frac{\mathrm{X} \sqrt{\omega}}{\sqrt{2 \mathrm{~K}_{1}}+e}-\frac{\mathrm{X} \sqrt{\omega}}{\sqrt{2 \mathrm{~K}_{2}}}\right]}\right.}{2+e^{-\frac{\mathrm{X} \sqrt{\omega}}{\sqrt{2 \mathrm{~K}_{2}}}}-e^{-\frac{\mathrm{X} \sqrt{\omega}}{\sqrt{2 \mathrm{~K}_{2}}}}} . .
$$

In order to handle this expression we may, as a close approximation, take $K_{1}$ equal to $K_{2}$, defining it in terms of the mean of the critical speeds of the two colours, $\frac{\omega_{R}+\omega_{G}}{2}$, by the relation

$$
\frac{\sqrt{2 \mathrm{~K}}}{\mathrm{X}}=\frac{\sqrt{\frac{\omega_{\mathrm{R}}+\omega_{\mathrm{G}}}{2}} \log e}{\log _{\delta_{\mathrm{B}}}^{2}}, . .
$$

We get then finally

$$
\log \delta_{\mathrm{B}}=\log 2-\sqrt{\frac{2 \omega_{\mathrm{M}}}{\omega_{\mathrm{R}}+\omega_{\mathrm{G}}}} \log \frac{2}{\delta_{\mathrm{B}}}
$$


$\omega_{\mathrm{M}}$ being the critical speed for the equality point $*$. Calculation of $\delta_{\mathrm{H}}$ therefore presupposes a knowledge of $\delta_{\mathrm{B}}$, and any error or variation in $\delta_{B}$ will be reflected in the value found for $\delta_{H} \dagger$. It must not be overlooked that while the brightness difference discrimination fraction has only one value, the hue fraction is a function of the colour difference constituting the ends of the scale; the larger the colour difference the smaller will this fraction be.

Exporimental values of $\delta_{\mathrm{H}}$ for the red-green comparison used in this test are given in the next section.

\section{Relative Values of Brightness and Hue Discrimination} Fractions under Steady and Fluctuating Conditions.

The question of crucial importance in the flicker photometer is not so much the actual values of the discriminating fractions, but whether their ratio is different for fluctuating and steady conditions. If this ratio is the same, then the mixture by rapid alternation possesses no advantago over any other form of mixture photometer, in which each field is diluted with light from the other until the colour difference is no longer troublesome.

In order to examine these ratios for both steady and fluctuating conditions, it was necessary to arrange for the measurement of both discrimination fractions with juxtaposad fields under steady conditions. This was simply arranged by viewing the mixture photometer glasses through a good bi-prism, size of field and brightness of retinal image being made the same as for the flicker photomoter work. The procedure in obtaining the brightness discrimination fraction was to use one light only, for which the green was taken, and turn the two opal glasses relative to each other until a difference of brightness was just noticeable between the two halves of the bi-prism, when the angle

- Fquation (11) can be used to calculate the critical speeds for all hue differences lying between any two for which $w_{x}$ has been determined, by nuting that halving the colour difference between the compared fields is equivalent to doubling the value of $\delta_{H}$, \&c. Critical speed hue difference curves when calculated in this wry have the general characteristics of the experimental one just published by Truland ("Appurent Brightness," Illuminating Engineering Society Convention, 1916). The critical speed for elimination of colour flicker between equally bright fields is a measure of their hue difference which may prove easier to determine than the present method of stepping off just noticeable differences. It should be possible to cunnect these critical speeds with the colour triangle.

$+\varepsilon_{\mathrm{n}}$ could also be obtained directly by a similar method to that used for $\delta_{B}$ by finding the critical speeds for various intermediate hue differences. 
was read. Readings were made in both directions of rotation, and half the difference taken as the least perceptible rotation, which was then translated into brightness discrimination fraction with the aid of the data of fig. 4 . The hue discrimination fraction was obtained in an exactly similar manner, both lights being used in this case, and the glasses being rotated until a difference of hue appeared between the two halves of the bi-prism.

These two steady observation discrimination fractions will be denoted as $\Delta_{\mathrm{B}}$ and $\Delta_{\mathrm{H}}$. Whether these fractions or the mean error of setting most nearly compare with the $\delta$ 's need not be discussed here, for we shall merely use the ratio of the two, which will be practically the same whichever measure of perception difference is taken.

The complete investigation of the various discrimination fractions and their relationship consists of five sets of measurements, which should be performed at one sitting in view of the apparent variability of some of them. These five measurements are as follows :-

1. Determination of $\delta_{\mathrm{B}}$.

2. Determination of equal brightness condition for two colours to be used for hue discrimination test.

3. Determination of $\delta_{\mathbf{H}}$ by critical speed measurements of two colours separately and together.

4. Determination of $\Delta_{\mathrm{B}}$.

5. Determination of $\Delta_{\mathrm{H}}$.

Three complete sets as thus outlined were carried through: two by the writer and a third by Mr. Kingsbury. The results are tabulated below :-

\begin{tabular}{|c|c|c|c|c|c|c|c|}
\hline & $\log i_{\mathrm{B}}$ & $\log \delta_{11}$ & $\frac{\delta_{\mathbf{H}}}{\delta_{\mathbf{B}}}$. & $\Delta_{\mathrm{B}}$ & $\Delta_{\mathbf{H}}$ & $-\frac{\Delta_{\mathbf{H}}}{\Delta_{\mathrm{B}}}$ & $\begin{array}{l}\frac{\delta_{K}}{\delta_{B}} \\
\Delta_{Y} \\
\vec{\Delta}_{B}\end{array}$ \\
\hline H. E. I. $\quad \ldots \ldots$ & $-3 \cdot 7$ & $-2 \cdot 79$ & $8 \cdot 1$ & 034 & $\cdot 034$ & $1 \cdot 0$ & $8 \cdot 1$ \\
\hline$,, ", \quad \ldots . . . .$. & -42 & -309 & $13 \cdot 0$ & $\cdot 025$ & $\cdot 03$ & $1 \cdot 2$ & $10 \cdot 8$ \\
\hline E. F. K. . ..... & $-3 \cdot 2$ & $-2 \cdot 07$ & $13 \cdot 5$ & $\cdot 044$ & $\cdot 08$ & $1 \cdot 8$ & $7 \cdot 4$ \\
\hline
\end{tabular}

The last column shows in a very striking manner to what the flicker photometer owes its peculiar applicability to colour difference work. In the confusion condition cuused by rapid alternation the appreciation of hue difference fails to 
keep pace with the appreciation of brightness difference. How necessary this eight- to ten-fold difference in the relative huo and brightness discriminations for steady and flicker conditions is, is shon $n$ graphically in fig. 7, where the upper dashed colour flicker curve (calculated by the aid of equation (11)), is the one which would condition the sensibility if the two discrimination fractions maintained the same relative values they have for steady juxtaposed fields. There would be no sensibility at all. This was further verified by attempting to operate the mixture photometer with the two glasses turned to the point of just noticeable hue difference. Either light source could then be placed practically anywhere on the photometer track without altering the brightness match.

This breakdown of hue discrimination is, of course, only another way of stating the common "explanation" of the flicker photometer, that " colour flicker ceases before brightness flicker." This is, however, the first time that definite measurements have been made of the visual constants whose values result in this condition. 'The explanation now needed is one step further back, namely, why does hue differenco discrimination fail in the ultimate receiving apparatus with rapidly alternating impressions?

It may be suggested, as a start toward an explanation, that colour discrimination is probably a later development than brightness discrimination, and that under conditions of confusion or stress the more primitive function maintains its characteristics with less impairment. The supposition may be hazarded that in general with the senses appreciation of quality differences is much easier disturbed than that of intensity differences. A close parallel to the action of the visual apparatus, as revealed by these measurements, is furnished by a flow-meter, such as a gas-meter, to which is attached an apparatus for indicating the kind of gas going through, the latter being conceived as a more complicated device requiring some time to respond. As long as only one kind of gas flows through the measurement of rate and the indication of composition are equally satisfactory. But let two different gases flow through in rapidly varying proportions. The precision of the gas-meter will be unchanged, but the composition indicator will be quite unable to respond to each momentary condition. 
9. Effect of Choice of Speed on Flicker Photometer Settings.

In the first theoretical paper the condition which must be satisfied when a setting is made on the flicker photometer was studied and found to be expressible by the equation

$$
2 \mathrm{I}_{1} e^{-\frac{\mathrm{x}_{1}}{\sqrt{2} \mathrm{~K}_{2}} \sqrt{\omega}}=2 \mathrm{I}_{2} e^{-\frac{\mathrm{x}_{2}}{\sqrt{2} \mathrm{~K}_{2}} \sqrt{\omega}} . \quad .
$$

The assumption was then made (assumption 3 of that paper), that the position of setting is independent of the speed, and the relationship of the flicker setting to the equal intensity and equal critical speed setting was developed for an assumed constant speed. In the light of the more recent work this assumption is no longer necessary, nor is it rigidly true. Inspection of fig. 7, for instance, shows that the bisector of the no-flicker region in the case of two different colours is inclined toward the colour of lower critical speed, so that the higher the working speed the more this will be favoured.

Introducing the complete expression for the diffusivity in terms of the critical speed-illumination relation, (12) becomes

$$
\log I_{1}-\frac{\sqrt{\omega} \log \frac{2}{\delta_{\mathrm{B}}}}{\sqrt{a_{1} \log I_{1}+b_{1}}}=\log I_{2}-\frac{\sqrt{\omega} \log \frac{2}{\delta_{\mathrm{B}}}}{\sqrt{a_{2} \log I_{2}+b_{2}}},
$$

in which the values of $I_{1}$ and $I_{2}$ which satisfy the equation are the ones which will be adjudged equal by the flicker photometer criterion, when the alterations occur at the speed $\omega$.

This expression shows that the point of setting is different for different speeds, and at the same time that the approach to equal brightness setting is closer the lower the speed. This emphasizes again the importance of avoiding all mechanical flicker and of selecting the speed for each colour difference.

Obvionsly $\omega$ should be the critical speed for the alpearance of colour ficker, which has been ealled $\omega_{\mathrm{M}}$ ibove. This is given in terms of $\delta_{\mathrm{B}}$ and $\delta_{\mathrm{B}}$ in equation (11), which transposed is,

$$
\omega_{\mathrm{M}}=\frac{\omega_{R}+\omega_{\mathrm{G}}}{2}\left(\frac{\log \frac{2}{\delta_{\mathrm{H}}}}{\log \frac{2}{\delta_{\mathrm{B}}}}\right)^{2} . . . .
$$

Substituting this in (13), the complete expression for the behaviour of the equal exposure flicker photometer, when Phil. Mag. S. 6. Vol. 33. No. 196. April 1917. 2 () 
operated at the point of just-disappearance of colour flicker, is

$$
\begin{aligned}
\log I_{1}- & \log \frac{2}{\delta_{\mathrm{H}}} \sqrt{\frac{\left(a_{1} \log \mathrm{I}_{1}+b_{1}\right)+\left(a_{2} \log \mathrm{I}_{2}+b_{2}\right)}{2\left(a_{1} \log \mathrm{I}_{1}+b_{1}\right)}} \\
= & \log \mathrm{I}_{2}-\log \frac{2}{\delta_{\mathrm{H}}} \sqrt{\frac{\left(a_{2} \log \mathrm{I}_{2}+b_{2}\right)+\left(a_{1} \log \mathrm{I}_{1}+b_{1}\right)}{2\left(a_{2} \log \mathrm{I}_{2}+b_{2}\right)}} .
\end{aligned}
$$

In making this substitution for $\delta_{\mathrm{H}}$ two assumptions figure : first, the one leading to equation (11), that the critical speed of the mixture of the two compared colours may be calculated to a close approximation by disregarding the different diffusivities of the two colours ; and second, that $\delta_{\mathrm{I}}$ is constant over the range of intensities in question. If these assumptions are true, then eyuation (14) states ( $\delta_{\mathrm{B}}$ having been found constant over wide range, as shown by the data of fig. 6) that the plot of $\omega_{M}$ against $\log I$ should be a straight line, lying helow the mein straight line given by the aritical frequencies for the two compared colours, and inclined to it at such an angle that both intersect the axis of abscisse at a common point.

Fig. 8.

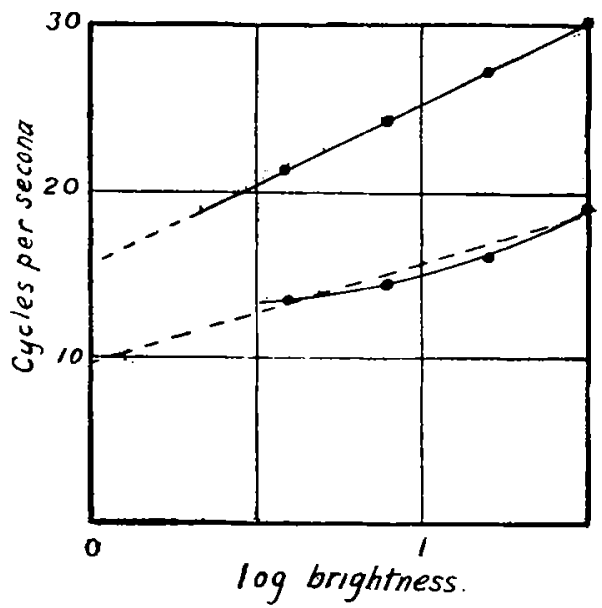

Upper points, mean critical frequencies for red and green light. Lower points, critical frequencies for red and green alternated against each other.

This relationship was tested out experimentally with the result shown in fig. 8, where the upper, straight line is 
the mean of the red and green light critical speed points, and the lower line is the critical speed plot for red and green alternated (the mean illumination being the same as for each alone) at their equality setting. The relationship between critical speed and log brightness shows a wellmarked deviation from the rectilinearity which wonld result were both the above assumptions correct. This is not surprising, for hue difference diserimination for steady observation raries quite rapidly with brightness, and in the flicker photometer the working speed at low intensities will be larger than that predicted from these assumptions owing to the pronounced la ${ }^{\circ}$ of one colour impression behind another * and the consequent lack of exact dove-tailing.

In spite of the failure of relation (14) to hold exactly, it gives the order of magnitude of $\omega_{M}$ so closely that the result of using (15) with $\delta_{\mathbf{H}}$ as a constant will be correct to within the probable errors of any experimental test. When used for calculations similar to those made in the first paper with $\omega$ assumed constant, the resultant predicted behaviour of the flicker photometer is quite similar, the deviations from the equal brimhtness position of setting being of course smaller, but decreasing with increased intensity as before. It may, in fact, he questioned whether the calculations made on the constant speed assumption do not more nearly represent the experimental conditions holding when the data with which they were compared were obtained, since some mechanical flicker undoubtedly prevented the nse of as low speeds as the colour differences used would warrant. It is to be hoped that data of this sort collected in future will be made with the polarization flicker photometer.

It may be noted, in passing, that the use of a variable instead of a constant speed in the calculation of the behaviour of the flicker photometer helps in the discussion of the unequal exposure instrument treated in the second paper. For by reducing the value of the constant $F$ in equation (14) of that paper the relative intensities necessary for a setting of the instrument are brought nearer together, in agreement with the experimental data.

\section{Summary.}

The results of this investigation may be summarized as follows :-

1. A polarization flicker photometer has been constructed, in which the transition from one field to the other is gradual

* See "Visual Diffusivity," Ires, Phil. May. Jan. 1917. 
and follows exactly the cosine curve assumed in the previous theoretical discussion of the flicker photometer.

2. By the use of this photometer it has been possible to verify in exact quantitative manner the relationships between relative intensity and absolute intensity of compared fields and critical speeds, as previously salculated from theory.

3. Measurements have been made of the brightness and hue discrimination fractions for both steady and fluctuating impressions.

4. It has been found that the applicability of the flicker photometer to colour difference comparisons is due to the failure of hue discrimination as compared with brightness discrimination under conditions of rapid fluctuation, and the amount of this failure has been determined by measurement.

5. A complete expression has been worked out for the behaviour of the flicker photometer in terms of the critical frequency-illumination relations for the two compared colours, and the hue discrimination fraction for fluctuating impressions.

I am greatly indebted to Mr. E. R. Morton for assistance in the construcion of the experimental apparatus and in making the readings, and also to $\mathrm{Mr}$. Kingsbury and Dr. Karrer for the series of observations made by them.

Physical Laboratory,

The United Gas Improvement Company, Philadelphia, Pa.

September 13th, 1916.

\section{Notices respecting New Books.}

Annuaire pour l'an 1917 publié par le Bureau des Longiturles. Paris: Gauthier-Villars. Price 2 fr. net.

W'TH very little delay appears this useful annual publication as though the world were at peace. Besides the usual astronomical tables there appear in this issue chapters on Metrology and Meteorology, besides special articles on (A) the Babyloninn calendar, (B) summer-time 1916 by .I. Renaud, (C) determination of the metre in terms of wave-lengths of light, by M. Hamy.

The article on summer-time, in particular, is of great historie interest in the summary it gives of the debates which took place in France and elsewhere prior to making this salutary change. 